\title{
Succination of Dihydrolipoyllysine Succinyltransferase (DLST) Exacerbates Mitochondrial ATP Deficiency in a Mouse Model of Leigh Syndrome
}

Gerardo G. Piroli ${ }^{1}$, Allison M. Manuel ${ }^{1}$, Holland H. Smith ${ }^{1}$, Richard S. McCain ${ }^{1}$, Michael D. Walla ${ }^{2}$, and Norma Frizzell ${ }^{1, *}$

${ }^{1}$ Department of Pharmacology, Physiology \& Neuroscience, School of Medicine, University of South Carolina, Columbia, SC 29209, USA,

${ }^{2}$ Mass Spectrometry Center, Department of Chemistry \& Biochemistry, University of South Carolina, Columbia, SC 29205, USA,

* To whom correspondence should be addressed: Norma Frizzell, Department of Pharmacology, Physiology \& Neuroscience, School of Medicine, University of South Carolina, 6439 Garners Ferry Road, Columbia, SC 29209, USA, Tel.: (803) 216-3521; Fax: (803) 216-3538; Email: norma.frizzell@uscmed.sc.edu 


\section{Summary}

The NDUFS4 knockout (KO) mouse phenotype resembles the human Complex I deficiency Leigh Syndrome. The irreversible succination of protein thiols by fumarate is increased in regions of the NDUFS4 KO brain affected by neurodegeneration, suggesting a mechanistic role in neurodegenerative decline. We report the identification of a novel succinated protein, dihydrolipoyllysine-residue succinyltransferase (DLST), a component of the $\alpha$-ketoglutarate dehydrogenase complex (KGDHC) of the tricarboxylic acid (TCA) cycle. Succination of DLST reduced KGDHC activity in the brainstem (BS) and olfactory bulb (OB) of KO mice. We further observed decreased mitochondrial substrate level phosphorylation, a TCA cycle reaction dependent on KGDHC derived succinyl-CoA, further aggravating the OXPHOS ATP deficit. Protein succinylation, an acylation modification that requires succinyl-CoA, was reduced in the KO mice. Our data demonstrate that the biochemical deficit extends beyond the Complex I assembly and energy defect, and functionally impairs multiple mitochondrial parameters to accelerate neuronal dysfunction. 


\section{Introduction}

Leigh syndrome (LS) is a mitochondrial disease caused predominantly by single gene defects in the oxidative phosphorylation (OXPHOS) machinery or the pyruvate dehydrogenase complex. It is characterized by bilateral necrotizing lesions of the basal ganglia and brainstem (BS), lactic acidosis, ataxia, intellectual retardation, seizures, and respiratory failure (Lake et al., 2016). Mutations in at least 23 genes that lead to mitochondrial Complex I deficiency are associated with LS (Lake et al., 2016). The most frequent cause is a mutation in NDUFS4, a gene that encodes the small assembly protein NADH dehydrogenase (ubiquinone) iron-sulfur protein 4 (Ndufs4) (Calvaruso et al., 2011; Ortigoza-Escobar et al., 2016).

The homozygous NDUFS4 knockout mouse model (Ndufs4 KO) recapitulates many biochemical and clinical aspects of LS, including lactic acidosis, BS degeneration, motor retardation and fatal respiratory failure at around 8 weeks after birth; the neuropathology also extends to the olfactory bulb (OB) and some cerebellar nuclei (Kruse et al., 2008; Quintana et al., 2010). Selective deletion of Ndufs4 in glutamatergic neurons leads to BS inflammation, motor and respiratory deficits and early death; whereas ablation of Ndufs4 in GABAergic neurons causes basal ganglia inflammation, hypothermia and severe epileptic seizures, highlighting combined contributions of different neuronal populations to the complex pathology of this model (Bolea et al., 2019). While the genetic defects underlying LS are well documented, the biochemical mechanisms linking the bioenergetic deficit to the onset of neurodegeneration remain ill defined. The presence of oxidative stress has been proposed as a contributor to the pathology in the brain of the Ndufs4 KO mouse. Augmented protein carbonylation was described in the OB (Quintana et al., 2010), but not in the motor cortex, of the KO mice (Felici et al., 2014); however the use of the antioxidant $\mathrm{N}$-acetylcysteine amide only modestly delayed the onset of motor symptoms (Liu et al., 2015). Increases in protein nitrotyrosine levels and 4-hydroxy-2-nonenal (HNE) protein adducts have been documented in total brain preparations, or in lesser-affected regions of the Ndufs4 KO mouse (Lee et al., 2019; Song et al., 2017; de Haas et al., 2017). However, we and others did not find increased HNE-protein conjugation in the pathologically lesioned BS (Kayser et al., 2016; Piroli et al., 2016). Specific measurement of the rate of $\mathrm{H}_{2} \mathrm{O}_{2}$ production by mitochondria respiring on malate and pyruvate demonstrated no difference in $\mathrm{H}_{2} \mathrm{O}_{2}$ production by the Ndufs4 KO versus WT (Jain et al., 2019). Since reductive stress is an established component of impaired OXPHOS (Titov et al., 2016), we had previously hypothesized that elevated $\mathrm{NADH} / \mathrm{NAD}^{+}$would interfere with the tricarboxylic acid (TCA) cycle, resulting in increased fumarate and protein succination, and that this contributes to the pathology observed in the Ndufs 4 KO brain. Succination is a post-translational modification of protein cysteine thiols due to their non-enzymatic and irreversible modification by fumarate, generating S-2-succinocysteine (2SC) (Figure S1A, Alderson et al., 2006; Frizzell et al., 2009; Merkley et al., 2014). Elevations in protein succination have been described in several tissues and conditions where fumarate is elevated, and is most frequently associated with reduced protein function (Kulkarni et al. 2019; Manuel et al., 2017; Piroli et al. 2014; Ternette et al. 2014). We were the first to demonstrate increased protein succination of select proteins in the most pathologically affected regions, e.g. the vestibular nucleus, of the Ndufs4 KO brain. We identified and confirmed the succination sites of mitochondrial voltage-dependent anion channels (VDAC) 1 and 2 (Piroli et al., 2016). Since an acidic environment favors this modification (Kulkarni et al., 2019); mitochondrial matrix 
acidification due to reduced electron transport chain activity may contribute to increased succination. In this study, we further pursued and identified dihydrolipoyllysine-residue succinyltransferase (DLST), a component of the $\alpha$-ketoglutarate dehydrogenase complex (KGDHC) of the TCA cycle. KGDHC is arguably the rate limiting step of the TCA cycle in the brain (Sheu and Blass, 1999), suggesting that deficient activity of this complex would have profound effects on the metabolism of the nervous system. Here, we report the striking functional impact of DLST succination on distinct mitochondrial metabolic processes. These results offer novel insight on the biochemical processes that exacerbate mitochondrial ATP deficits beyond the existing Complex I bioenergetic defect. 


\section{Results}

Dihydrolipoyllysine-residue succinyltransferase is succinated in the Ndufs4 KO mouse brain

In order to identify new target(s) of succination in the brainstem (BS) and olfactory bulb (OB) of the Ndufs4 KO mouse, we focused our attention on a band that was immunoreactive with anti-2SC antibody only in the KO tissues; this band was located at $\sim 48-50 \mathrm{kDa}$, immediately below the succinated tubulin band (Piroli et al., 2016). Using high speed centrifugation pellets, we confirmed that this succinated band was present in KO but not in WT mice BS preparations (Figure 1A, arrow in 2SC panel), and was further enriched following depletion of tubulin (Figure S1B, arrow in 2SC panel). To further characterize this protein we isolated gliosomes and synaptosomes from WT and Ndufs4 KO mouse BS; this gradient preparation generates a loose pellet at the bottom of the centrifugation tube that was also analyzed. Glial fibrillary acidic protein (GFAP), an astrocytic marker, was enriched in the gliosomes, whereas the presence of the neuronal presynaptic marker synaptophysin was prominent in the synaptosomes (Figure 1B, GFAP and synaptophysin panels). The outer mitochondrial membrane marker VDAC2 was present in the homogenate and the synaptosomes, and further enriched in the pellet fractions (Figure 1B, VDAC2 panel). Overall, the pellets were rich in mitochondria but devoid of cytosolic, synaptic and astroglial markers. After probing with anti-2SC antibody, the succinated band at $\sim 48-50 \mathrm{kDa}$ was clearly detectable in the pellet fraction of Ndufs4 KO but not in WT mice (Figure 1B, arrow in 2SC panel). Tubulin depletion facilitated this visualization in the pellets (Figure 1B, $\alpha$-tubulin panel); in all the other fractions, the abundant presence of succinated tubulin both in WT and KO preparations overwhelms the detection of this $\sim 48-50 \mathrm{kDa}$ band (Figure 1B, 2SC panel). Similar results were obtained with preparations from the cerebellum, another affected brain region in Ndufs4 KO mice (Figure S1C). To identify this succinated protein, the pellet fractions were further separated by SDS-PAGE, with parallel lanes used for anti-2SC immunoblotting or Coomassie staining, prior to band excision and LC-MS/MS mass spectrometry analysis. This proteomic strategy identified the mitochondrial form of the dihydrolipoyllysine-residue succinyltransferase (DLST) component of the KGDHC, whose isoelectric point (pl) 5.98 corresponded to a previously unidentified train of succinated spots on 2D-separated immunoblots (Piroli et al. 2016). The KGDHC comprises several copies of three subunits: $\alpha$-ketoglutarate dehydrogenase (KGDH, E1k), DLST (E2k), and dihydrolipoamide dehydrogenase (DLD, E3) (Reed and Oliver, 1982), with E1k and E2k being specific for this complex and E3 shared with other $\alpha$-ketoacid dehydrogenases (Reed, 1974). Supplemental Table 1 summarizes the DLST peptides confirmed by mass spectrometry, with a representative validated peptide (XCorr $(+2)=3.94)$ NVETM ${ }^{\text {OX }}$ NYADIER $\left([\mathrm{M}+2 \mathrm{H}]^{2+}\right.$ : 735.8322) shown in Figure 1D. To further confirm DLST as the succinated protein identified, we analyzed total homogenates and purified mitochondrial fractions from WT and Ndufs 4 KO mice by immunoblot. Figure $1 \mathrm{C}$ shows that BS homogenates from KO and WT mice did not visibly differ in terms of protein succination at a low exposure (2SC panel, homogenate lanes); only the pronounced succinated tubulin band at $\sim 50-55 \mathrm{kDa}$ was present (Piroli et al., 2014; Piroli et al., 2016). By depleting tubulin, mitochondrial purification allowed for a better resolution of the band at $\sim 48-50 \mathrm{kDa}$; a distinct band with increased succination in the KO was present (2SC panel, mitochondria lanes, black arrow), confirming the observations in Figure 1B. The additional succinated band at $\sim 30-32 \mathrm{kDa}$ had been previously identified as VDAC1 and 2 (Piroli et al., 2016). After stripping, a specific anti-DLST antibody showed similar levels of this protein in both WT and Ndufs4 KO mitochondria 
(DLST panel, mitochondria lanes), and the DLST bands overlapped precisely with the succinated bands at $\sim 48-50 \mathrm{kDa}$ (2SC panel, mitochondria lanes).

To specifically confirm if the DLST identification corresponded to the train of spots previously observed on 2D immunoblots (Piroli et al. 2016), we performed a separation of BS proteins by 2D-gel electrophoresis, where the first dimension was isoelectric focusing in a $\mathrm{pH}$ gradient of 4 to 7 . This procedure allowed the separation of the tubulin isoforms from other proteins with similar MW but different pl. As shown in Figure 1D (2SC panels), succinated tubulins appeared as an intense spot at $\sim 50$ $55 \mathrm{kDa}$ that extends across the range of pl 4.7-5.4 both in WT and KO preparations, as previously reported (Piroli et al., 2014; Piroli et al., 2016). We focused our attention on the train of succinated spots at $\sim 48-50$ $\mathrm{kDa}$ (highlighted with a rectangle). In the KO preparation several spots were present in this area (Figure 1D, 2SC KO panel), whereas in the WT preparation only one spot was present (Figure 1D, 2SC WT panel). After stripping, the same blots were incubated with a DLST specific antibody and a train of spots were detected both in the WT and KO blots (Figure 1D, DLST panels). In the WT blots, the four spots detected by anti-DLST do not overlap with any succinated spot (Figure 1D, WT DLST and 2SC panel). In contrast, three succinated spots co-localize with three of the spots in the DLST blot in KO preparations (Figure 1D, overlap marked by arrows in KO 2SC and DLST panels). Since the isoelectric focusing can contribute to uneven protein loading, we further confirmed that total DLST protein levels do not change in the BS between genotypes, relative to $\alpha$-tubulin expression, as shown in Figure $1 \mathrm{E}$ and $\mathrm{F}$. In summary, we confirmed that specific isoforms of DLST are uniquely succinated in the BS of Ndufs4 KO mice compared to WT mice, and the total protein level of DLST is unchanged.

Succination reduces the activity of the $\alpha$-ketoglutarate dehydrogenase complex

We hypothesized that DLST succination driven by increases in fumarate content might affect the functionality of the KGDHC, since components of this complex are susceptible to oxidative modification (Chinopoulos et al., 1999; Humphries and Szweda, 1998). To test this, we first confirmed increased fumarate concentration in the OB of the Ndufs4 KO mouse $196.7 \%$ greater than in WT OB, $p<0.001$, Figure $2 A)$. We then measured KGDHC activity in both the BS and OB of WT and Ndufs 4 KO mice, and found that in both brain regions it was significantly lower in the KO mice $(24.2$ and $31.2 \%$ decrease in BS and OB, $\mathrm{p}<0.05$ and 0.01 respectively, Figures $2 \mathrm{~B}$ and $2 \mathrm{C}$ ). Fumarate mediated reduction in the activity of the dehydrogenase was also confirmed in vitro following incubation of an isolated KGDHC standard with a range of dimethyl fumarate (DMF) concentrations, as treatment with this reactive fumarate ester also results in protein succination (Piroli et al., 2019). Figure 2D demonstrates that DMF treatment caused a dose-dependent decrease in the activity of the complex. Furthermore, we confirmed decreased KGDHC activity in N1E-115 neurons that were incubated with DMF for $18 \mathrm{~h}$ (Figure 2E). When we replaced DMF with dimethyl succinate, a DMF analog that does not modify cysteine residues due to the lack of the double bond, there was no loss of KGDHC activity (Figure 2E). Interestingly, increased succination of a band at $\sim 48-50 \mathrm{kDa}$ was observed in N1E-115 neurons after incubation with 50 and $100 \mu \mathrm{M}$ DMF (Figure $2 \mathrm{~F}$, arrow in $2 \mathrm{SC}$ panel), and the band co-localized with DLST immunolabeling (Figure 2F, DLST panel). The specific reduction in KGDHC functionality following fumarate ester treatment supports the demonstration of reduced KGDHC activity in both the $\mathrm{OB}$ and $\mathrm{BS}$, where endogenously produced fumarate, and the specific succination of DLST are elevated. 
Substrate level phosphorylation is decreased in the Ndufs4 KO mouse brain.

Since decreased KGDHC activity would lead to reduced formation of succinyl CoA, this in turn would likely decrease the conversion of succinyl-CoA into succinate in the TCA cycle. This step is catalyzed by the enzyme succinyl-CoA ligase, which is responsible for the synthesis of GTP or ATP by substrate level phosphorylation (SLP). In rodents and human brain, succinyl-CoA ligase preferentially produces ATP, whereas in anabolic tissues the main product is GTP (Lambeth et al., 2004; Ostergaard, 2008). We measured total and SLP-linked ATP synthesis in mitochondria isolated from both OB and BS of WT and Ndufs4 KO mice. Under the conditions used, total ATP synthesis represents the sum of ATP production by OXPHOS and SLP, and the residual ATP synthesis in the presence of the ATP synthase inhibitor oligomycin represents SLP (Komlódi and Tretter, 2017). Using $\alpha$-ketoglutarate as a substrate, both total ATP synthesis and SLP were decreased in the OB of the KO mice by $42.5 \%(p<0.05)$ and $48.3 \%(p<0.05)$, respectively (Figure $3 \mathrm{~A}$ ). These data demonstrate that, in addition to the Complex I defect in OXPHOS derived ATP production, the generation of TCA cycle derived intramitochondrial ATP is also compromised. In contrast, we observed no significant differences in total ATP synthesis and SLP in the BS of Ndufs4 KO vs. WT mice (Figure 3B). This may reflect the heterogeneity of the total BS mitochondrial preparation, where select nuclei appear to be more affected by pathology than others. When succinate was used as a respiratory substrate, no differences in total ATP synthesis were observed for OB (Figure 3C) and BS (Figure 3D). As expected, succinate did not support SLP irrespective of the genotype and brain region (Figure $3 \mathrm{C}$ and D) (Komlódi and Tretter, 2017).

Succinylation of mitochondrial proteins is reduced in the Ndufs4 KO mouse brain

Decreased synthesis of succinyl-CoA could also be expected to reduce protein succinylation, a distinct acyl CoA derived modification (Zhang et al. 2011), in brain regions of the Ndufs4 KO mouse. Protein succinylation has been implicated in modulating the activity of mitochondrial enzymes (Gibson et al., 2015; Park et al., 2013). DLST can act as a protein succinyl transferase (Gibson et al., 2015), therefore the succination of DLST could contribute directly to decreased protein succinylation. As shown in Figure 4A, the intensity of protein succinylation was markedly decreased in the mitochondrial fractions from BS of Ndufs4 KO mice compared to their corresponding WT littermates. The quantification of the reduction observed in the $\mathrm{KO}$ for the most intense bands is shown in Figure 4B, demonstrating greater than $50 \%$ reductions in succinylation levels for these proteins. In contrast, total levels of acetyl CoA derived mitochondrial protein acetylation (Figure $4 \mathrm{C}$ ), demonstrated an increase for select proteins in the Ndufs4 KO versus the WT (Figure 4D, quantification for several acetylated protein bands was performed). A parallel analysis of succinated proteins demonstrated that a band at $\sim 48-50 \mathrm{kDa}$ was present in these mitochondrial fractions (Supplementary Figure 2), confirming the results in Figures $1 B$ and $1 C$ that DLST is succinated. In summary, we confirmed that impaired DLST function results in reductions in succinyl CoA production that is associated with decreased protein succinylation. In contrast, protein acetylation is increased as acetyl CoA production occurs prior to the defective KGDHC in Ndufs4 KO. 


\section{Discussion}

The results of the current study mechanistically demonstrate that DLST, a component of the KGDHC, is succinated by fumarate in the BS and OB of the Ndufs 4 KO mouse, two brain regions where the most profound pathological changes were described in this LS model (Quintana et al., 2010). Consistent with DLST succination, we observed decreased activity of the KGDHC in these same regions of the Ndufs4 KO mouse brain. These important results link an OXPHOS genetic defect to impaired TCA function that further impacts succinyl-CoA production, a TCA intermediate necessary for SLP (Kiss et al., 2013; Komlódi and Tretter, 2017) and succinylation reactions (Yang and Gibson, 2019). While variable redox changes impact TCA cycle activity depending on energy status of the cell, protein succination represents a static event that contributes to functional decline of the affected proteins, elucidating an unrecognized pathological contributor in Complex I deficiency.

The role of KGDHC deficiency in neurodegeneration has been extensively studied in the preclinical setting, including the genetic modulation of DLD and DLST; in both cases the KO mice were not viable (Johnson et al., 1997; Yang et al., 2009). DLST ${ }^{+/-}$and $\mathrm{DLD}^{+/-}$do not show apparent symptoms, but are susceptible to mitochondrial toxins used to replicate neurodegenerative diseases (Klivenyi et al., 2004; Yang et al., 2009). Pharmacological inhibition of KGDHC activity in vitro alters mitochondrial morphology and increases fission and mitophagy (Banerjee et al., 2016). In the clinical setting, patients with mutations in the LIPT1/2 genes that encode a lipoyltransferase necessary for DLST lipoylation develop LS-like encephalopathies (Habarou et al., 2017; Stowe et al., 2018), supporting a role for defective KGDHC components in neuropathology. Human brain samples from patients with Alzheimer's disease (AD) showed reduced KGDHC activity (Butterworth and Besnard, 1990; Gibson et al., 1988). Interestingly, the overall decrease of KGDHC activity in $A D$ is greater than the reduction in the content of the individual components of the complex, suggesting that other regulatory factors, potentially post-translational modification, play a role in the deficiency (Mastrogiacomo et al., 1996). As we found similar levels of DLST in WT and Ndufs4 KO mice, we suggest succination-driven reductions in KGDHC activity as a contributor to the neurodegeneration observed in this LS model. Interestingly, the degree of KGDHC inhibition in the brains of Ndufs $4 \mathrm{KO}$ mice is similar to that reported using pharmacological inhibitors of the complex to impair mitochondrial function (Banerjee et al., 2016).

Previous reports on ATP synthesis in the Ndufs4 KO mouse showed vary depending on the tissue analyzed, with no differences in total cellular ATP content in immortalized fibroblasts (Valsecchi et al., 2012) and skeletal muscle (Alam et al., 2015; Kruse et al., 2008; Terburgh et al., 2019) compared to WT mice. However, maximal mitochondrial ATP production in the whole brain is slightly decreased in Ndufs4 KO versus WT mice (Manjeri et al., 2016). In isolated permeabilized neurons and astrocytes from mice with a spontaneous mutation leading to disruption of NDUFS4, ATP synthesis driven by substrates that fuel $\mathrm{Cl}$ was also slightly decreased (Bird et al., 2014). Here we describe that both total and SLP-linked ATP synthesis in mitochondria isolated from the OB of the Ndufs4 KO mouse were decreased when $\alpha$ ketoglutarate, a fuel that supports SLP, was used. A previous study using brain mitochondria from $\mathrm{DLD}^{+/}$ and $\mathrm{DLST}^{+/}$mice showed diminished mitochondrial ATP efflux with fuel combinations that support SLP compared to WT mitochondria, and these mice also had a $20-48 \%$ decrease in KGDHC activity, which is consistent with our findings (Kiss et al., 2013). Remarkably, patients with deficiencies in the ATP-linked 
isoform of succinyl-CoA ligase, the TCA enzyme responsible for SLP, develop a LS-like syndrome (Ostergaard, 2008).

Supplementation with dimethyl $\alpha$-ketoglutarate (DMKG), a cell permeable form of $\alpha$-ketoglutarate, was successfully used to increase the lifespan of Ndufs4 KO mice and delay neurological symptoms occurrence (Lee et al., 2019). The mechanism proposed was the suppression of hypoxic signaling through decreased HIF1 $\alpha$ levels, which is in accordance with recent data showing that genetic activation of the hypoxia response is insufficient and even detrimental to rescue the disease (Jain et al., 2019). While DMKG may serve as a source of $\mathrm{NADH}$ for $\mathrm{Cl}$, this benefit would be limited in this $\mathrm{Cl}$-deficient mouse; this is in line with our observed decrease in total and SLP-linked ATP synthesis when $\alpha$-ketoglutarate was used as respiratory substrate. In fact, the energetic deficit due to reduced SLP-linked ATP synthesis that we describe might further compound not only the genetic OXPHOS deficiency, but also a proposed dysfunctional ATP/ADP translocation due to VDAC succination in this model (Piroli et al., 2016).

As succinyl-CoA is required not only for SLP, but also for protein succinylation (Zhang et al., 2011), we predicted a decrease in this post-translational modification in the brain of the Ndufs4 KO mouse. Alterations in succinylation have not previously been documented in mitochondrial diseases, and we demonstrate a profound decrease in global mitochondrial succinylation. In contrast to succination, succinylation is a reversible modification (by SIRT enzymes) due to the reaction between succinyl-CoA and lysine residues (Yang and Gibson, 2019). The KGDHC was shown to succinylate proteins in vitro more efficiently than free succinyl-CoA, probably due to the succinyl transferase activity of the E2k component (Gibson et al., 2015). In primary neurons, KGDHC inhibitors reduce succinylation of cytosolic and mitochondrial proteins (Gibson et al., 2015), in agreement with our observed decrease in protein succinylation in the BS mitochondria of the Ndufs4 KO. In addition, hypoxia has been recently shown to prolong lifespan and reverse the brain lesions of the Ndufs4 KO mouse (Jain et al., 2016; Ferrari et al., 2017). Reduction of oxygen content in the air normalizes the oxygen concentration in the brain of the Ndufs4 KO mouse, as it is hyperoxic, to levels similar to those found in WT littermates breathing normal air (Jain et al., 2019). Interestingly, in vitro studies with N2a neural cells showed that hypoxia leads to increased mitochondrial protein succinylation (Chen et al 2017). We hypothesize that the reduced availability of succinyl-CoA is augmented by hyperoxia in the brain tissue, leading to the decreased protein succinylation observed in the Ndufs4 KO mouse.

In summary, we demonstrate that fumarate driven succination of DLST irreversibly reduces the activity of the KGDHC in the pathologically affected brain mitochondria of the Ndufs4 KO mouse. This persistent deficit worsens the mitochondrial OXPHOS derived ATP deficit by limiting SLP. These data mechanistically demonstrate that a Complex I deficit results in distinct metabolite alterations that functionally impair the TCA cycle, and provide a novel biochemical explanation for the basis of mitochondrial pathophysiology. 
bioRxiv preprint doi: https://doi.org/10.1101/2020.01.09.900514; this version posted January 9,2020 . The copyright holder for this preprint

(which was not certified by peer review) is the author/funder, who has granted bioRxiv a license to display the preprint in perpetuity. It is made available under aCC-BY-NC-ND 4.0 International license.

\section{Author Contributions}

G.G.P and N.F. designed the research. G.G.P., A.M.M., H.H.S., R.S.M. and M.D.W. performed experiments. G.G.P., A.M.M., H.H.S., M.D.W. and N.F. analyzed data. G.G.P., A.M.M., and N.F. wrote the paper.

\section{Acknowledgements}

This work was supported by the University of South Carolina Research Foundation ASPIRE-I award and the National Institutes of Health (R01 NS092938, R03 HD077187, R56 DK105087, F31 DK108559). 


\section{References}

Alam, M.T., Manjeri, G.R., Rodenburg, R.J., Smeitink, J.A., Notebaart, R.A., Huynen, M., Willems, P.H., and Koopman, W.J. (2015). Skeletal muscle mitochondria of NDUFS4-/- mice display normal maximal pyruvate oxidation and ATP production. Biochim. Biophys. Acta 1847, 526-533.

Alderson, N.L., Wang, Y., Blatnik, M., Frizzell, N., Walla, M.D., Lyons, T.J., Alt, N., Carson, J.A., Nagai, R., Thorpe, S.R., et al. (2006). S-(2-Succinyl)cysteine: A novel chemical modification of tissue proteins by a Krebs cycle intermediate. Arch. Biochem. Biophys. 450, 1-8.

Banerjee, K., Munshi, S., Xu, H., Frank, D.E., Chen, H.L., Chu, C.T., Yang, J., Cho, S., Kagan, V.E., Denton, T.T., et al. (2016). Mild mitochondrial metabolic deficits by $\alpha$-ketoglutarate dehydrogenase inhibition cause prominent changes in intracellular autophagic signaling: Potential role in the pathobiology of Alzheimer's disease. Neurochem. Int. 96, 32-45.

Bird, M.J., Wijeyeratne, X.W., Komen, J.C., Laskowski, A., Ryan, M.T., Thorburn, D.R., and Frazier, A.E. (2014). Neuronal and astrocyte dysfunction diverges from embryonic fibroblasts in the Ndufs4fky/fky mouse. Biosci. Rep. 34:e00151.

Bolea, I., Gella, A., Sanz, E., Prada-Dacasa, P., Menardy, F., Bard, A.M., Machuca-Márquez, P., Eraso-Pichot, A., Mòdol-Caballero, G., Navarro, X., et al. (2019). Defined neuronal populations drive fatal phenotype in a mouse model of Leigh syndrome. eLife 8:e47163.

Butterworth, R.F., and Besnard, A.M. (1990). Thiamine-dependent enzyme changes in temporal cortex of patients with Alzheimer's disease. Metab. Brain. Dis. 5, 179-184.

Calvaruso, M.A., Willems, P., van den Brand, M., Valsecchi, F., Kruse, S., Palmiter, R., Smeitink, J., and Nijtmans, L. (2011). Mitochondrial complex III stabilizes complex I in the absence of NDUFS4 to provide partial activity. Hum. Mol. Genet. 21:115-120.

Carney, K.E., Milanese, M., van Nierop, P., Li, K.W., Oliet, S.H., Smit, A.B., Bonanno, G., and Verheijen, M.H. (2014). Proteomic analysis of gliosomes from mouse brain: identification and investigation of glial membrane proteins. J. Proteome Res. 13, 5918- 5927.

Chen, H., Xu, H., Potash, S., Starkov, A., Belousov, V.V., Bilan, D.S., Denton, T.T., and Gibson, G.E. (2017). Mild metabolic perturbations alter succinylation of mitochondrial proteins. J. Neurosci. Res. 95, 22442252.

Chinopoulos, C., Tretter, L., and Adam-Vizi, V. (1999). Depolarization of in situ mitochondria due to hydrogen peroxide-induced oxidative stress in nerve terminals: inhibition of alpha-ketoglutarate dehydrogenase. J Neurochem. 73, 220-228.

de Haas, R., Das, D., Garanto, A., Renkema, H.G., Greupink, R., van den Broek, P., Pertijs, J., Collin, R.W.J., Willems, P., Beyrath, J.,et al. (2017). Therapeutic effects of the mitochondrial ROS-redox modulator KH176 in a mammalian model of Leigh Disease. Sci. Rep. 7:11733. 
Felici, R., Cavone, L., Lapucci, A., Guasti, D., Bani, D., and Chiarugi, A. (2014). PARP inhibition delays progression of mitochondrial encephalopathy in mice. Neurotherapeutics 11, 651-664.

Ferrari, M., Jain, I.H., Goldberger, O., Rezoagli, E., Thoonen, R., Cheng, K.H., Sosnovik, D.E., ScherrerCrosbie, M., Mootha, V.K., and Zapol, W.M. (2017). Hypoxia treatment reverses neurodegenerative disease in a mouse model of Leigh syndrome. Proc. Natl. Acad. Sci. U S A. 114, E4241-E4250.

Frizzell, N., Rajesh, M., Jepson, M.J., Nagai, R., Carson, J.A., Thorpe, S.R., and Baynes, J.W. (2009). Succination of thiol groups in adipose tissue proteins in diabetes: Succination inhibits polymerization and secretion of adiponectin. J. Biol. Chem. 284, 25772-25781.

Gibson, G.E., Sheu, K.F.R., Blass, J.P., Baker, A., Carlson, K.C., Harding, B., and Perrino, P. (1988). Reduced activities of thiamine-dependent enzymes in the brains and peripheral tissues of patients with Alzheimer's disease. Arch. Neurol. 45, 836-840.

Gibson, G.E., Xu, H., Chen, H.L., Chen, W., Denton, T.T., and Zhang, S. (2015). Alpha-ketoglutarate dehydrogenase complex-dependent succinylation of proteins in neurons and neuronal cell lines. J. Neurochem. 134, 86-96.

Habarou, F., Hamel, Y., Haack, T.B., Feichtinger, R.G., Lebigot, E., Marquardt, I., Busiah, K., Laroche, C., Madrange, M., Grisel, C., et al. (2017). Biallelic mutations in LIPT2 cause a mitochondrial lipoylation defect associated with severe neonatal encephalopathy. Am. J. Hum. Genet. 101, 283-290.

Humphries, K.M., and Szweda, L.I. (1998). Selective inactivation of $\alpha$-ketoglutarate dehydrogenase and pyruvate dehydrogenase: Reaction of lipoic acid with 4-hydroxy-2-nonenal. Biochemistry 37, 1583515841.

Jain, I.H., Zazzeron, L., Goldberger, O., Marutani, E., Wojtkiewicz, G.R., Ast ,T,, Wang, H., Schleifer, G., Stepanova, A., Brepoels, K., et al. (2019). Leigh Syndrome mouse model can be rescued by interventions that normalize brain hyperoxia, but not HIF activation. Cell Metab. 30, 824-832.

Jain, I.H., Zazzeron, L., Goli, R., Alexa, K., Schatzman-Bone, S., Dhillon, H., Goldberger, O., Peng, J., Shalem, O., Sanjana, N.E., et al. (2016). Hypoxia as a therapy for mitochondrial disease. Science 352, 54-61.

Johnson, M.T., Yang, H.S., Magnuson, T., and Patel, M.S. (1997). Targeted disruption of the murine dihydrolipoamide dehydrogenase gene (DId) results in perigastrulation lethality. Proc. Natl. Acad. Sci. U S A. $94,14512-14517$.

Kayser, E.B., Sedensky, M.M., and Morgan, P.G. (2016). Region-specific defects of respiratory capacities in the Ndufs4(KO) mouse brain. PLoS One 11:e0148219.

Kiss, G., Konrad, C., Doczi, J., Starkov, A.A., Kawamata, H., Manfredi, G., Zhang, S.F., Gibson, G.E., Beal, M.F., Adam-Vizi, V., et al. (2013). The negative impact of $\alpha$-ketoglutarate dehydrogenase complex deficiency on matrix substrate-level phosphorylation. FASEB J. 27, 2392-2406. 
Klivenyi, P., Starkov, A.A., Calingasan, N.Y., Gardian, G., Browne, S.E., Yang, L., Bubber, P., Gibson, G.E., Patel, M.S., and Beal, M.F. (2004). Mice deficient in dihydrolipoamide dehydrogenase show increased vulnerability to MPTP, malonate and 3-nitropropionic acid neurotoxicity. J. Neurochem. 88, 1352-1360.

Komlódi, T., and Tretter, L. (2017). Methylene blue stimulates substrate-level phosphorylation catalyzed by succinyl-CoA ligase in the citric acid cycle. Neuropharmacology 123, 287-298.

Kruse, S.E., Watt, W.C., Marcinek, D.J., Kapur, R.P., Schenkman, K.A., and Palmiter, R.D. (2008). Mice with mitochondrial complex I deficiency develop a fatal encephalomyopathy. Cell Metab. 7, 312-320.

Kulkarni, R.A., Bak, D.W., Wei, D., Bergholtz, S.E., Briney, C.A., Shrimp, J.H., Alpsoy, A., Thorpe, A.L., Bavari, A.E., Crooks, D.R., et al. (2019). A chemoproteomic portrait of the oncometabolite fumarate. Nat. Chem. Biol. 15, 391-400.

Lake, N.J., Compton, A.G., Rahman, S., and Thorburn, D.R. (2016). Leigh syndrome: One disorder, more than 75 monogenic causes. Ann. Neurol. 79, 190-203.

Lambeth, D.O., Tews, K.N., Adkins, S., Frohlich, D., and Milavetz, B.I. (2004). Expression of two succinylCoA synthetases with different nucleotide specificities in mammalian tissues. J. Biol. Chem. 279, 3662136624.

Lee, C.F., Caudal, A., Abell, L., Nagana Gowda, G.A., and Tian, R. (2019). Targeting NAD ${ }^{+}$metabolism as interventions for mitochondrial disease. Sci. Rep. 9:3073.

Liu, L., Zhang, K., Sandoval, H., Yamamoto, S., Jaiswal, M., Sanz, E., Li, Z., Hui, J., Graham, B.H., Quintana, A., et al. (2015). Glial lipid droplets and ROS induced by mitochondrial defects promote neurodegeneration. Cell 160, 177-190.

Lowry, O. H., Rosenbrough, N. J., Farr, A. L. and Randall, R. J. (1951). Protein measurement with the Folin phenol reagent. J. Biol. Chem. 193, 265-275.

Manjeri, G.R., Rodenburg, R.J., Blanchet, L., Roelofs, S., Nijtmans, L.G., Smeitink, J.A., Driessen, J.J., Koopman, W.J., and Willems, P.H. (2016). Increased mitochondrial ATP production capacity in brain of healthy mice and a mouse model of isolated complex I deficiency after isoflurane anesthesia. J. Inherit. Metab. Dis. 39, 59-65.

Manuel, A.M., Walla, M.D., Faccenda, A., Martin, S.L., Tanis, R.M., Piroli, G.G., Adam, J., Kantor, B., Mutus, B., Townsend, D.M., et al. (2017). Succination of protein disulfide isomerase links mitochondrial stress and endoplasmic reticulum stress in the adipocyte during diabetes. Antioxid. Redox Signal. 27, 1281-1296.

Mastrogiacomo, F., Lindsay, J.G., Bettendorff, L., Rice, J., and Kish, S.J. (1996). Brain protein and $\alpha$ ketoglutarate dehydrogenase complex activity in Alzheimer's disease. Ann. Neurol. 39, 592-598.

Merkley, E.D., Metz, T.O., Smith, R.D., Baynes, J.W., and Frizzell, N. (2014). The succinated proteome. Mass Spectrom. Rev. 33, 98-109. 
Nagai, R., Brock, J. W., Blatnik, M., Baatz, J. E., Bethard, J., Walla, M. D., Thorpe, S. R., Baynes, J. W., and Frizzell, N. (2007). Succination of protein thiols during adipocyte maturation: a biomarker of mitochondrial stress. J. Biol. Chem. 282, 34219-34228.

Ortigoza-Escobar, J.D., Oyarzabal, A., Montero, R., Artuch, R., Jou, C., Jiménez, C., Gort, L., Briones, P., Muchart, J., López-Gallardo, E., et al. (2016). Ndufs4 related Leigh syndrome: A case report and review of the literature. Mitochondrion 28, 73-78.

Ostergaard, E. (2008). Disorders caused by deficiency of succinate-CoA ligase. J. Inherit. Metab. Dis. 31, 226-229.

Park, J., Chen, Y., Tishkoff, D.X., Peng, C., Tan, M., Dai, L., Xie, Z., Zhang, Y., Zwaans, B.M.M., Skinner, M.E., Lombard, D.B., and Zhao, Y. (2013). SIRT5-mediated lysine desuccinylation impacts diverse metabolic pathways. Mol. Cell 50, 919-930.

Piroli, G.G., Jepson, M.J., Manuel, A.M., Walla, M.D., Brock, J.W.C., Rajesh, M.P., Tanis, R.M., Cotham, W.E., and Frizzell, N. (2014). Identification of protein succination as a novel modification of tubulin. Biochem. J. 462, 231-245.

Piroli, G.G., Manuel, A.M., Clapper, A.C., Walla, M.D., Baatz, J.E., Palmiter, R.D., Quintana, A., and Frizzell, N. (2016). Succination is increased on select proteins in the brainstem of the NADH dehydrogenase (ubiquinone) Fe-S protein 4 (Ndufs4) knockout mouse, a model of Leigh Syndrome. Mol. Cell. Proteomics $15,445-461$.

Piroli, G.G., Manuel, A.M., Patel, T., Walla, M.D., Shi, L., Lanci, S.A., Wang, J., Galloway, A., Ortinski, P.I., Smith, D.S., et al. (2019). Identification of novel protein targets of dimethyl fumarate modification in neurons and astrocytes reveals actions independent of Nrf2 stabilization. Mol. Cell. Proteomics 18, 504519.

Quintana, A., Kruse, S.E., Kapur, R.P., Sanz, E., and Palmiter, R.D. (2010). Complex I deficiency due to loss of Ndufs 4 in the brain results in progressive encephalopathy resembling Leigh syndrome. Proc. Natl. Acad. Sci. U S A. 107, 10996-11001.

Reed, L.J. (1974). Multienzyme complexes. Acc. Chem. Res. 7, 40-46.

Reed, L.J., and Oliver, R.M. (1982). Structure-function relationships in pyruvate and $\alpha$-ketoglutarate dehydrogenase complexes. Adv. Exp. Med. Biol. 148, 231-241.

Schneider, C.A., Rasband, W.S., and Eliceiri, K.W. (2012). NIH Image to ImageJ: 25 years of image analysis. Nat. Methods 9, 671-675.

Sheu, K.F.R., and Blass, J.P. (1999). The $\alpha$-ketoglutarate dehydrogenase complex. Ann. N Y Acad. Sci. 893, 61-78.

Song, L., Yu, A., Murray, K., and Cortopassi, G. (2017). Bipolar cell reduction precedes retinal ganglion neuron loss in a complex 1 knockout mouse model. Brain Res. 1657, 232-244. 
Stowe, R.C., Sun, Q., Elsea, S.H., and Scaglia, F. (2018). LIPT1 deficiency presenting as early infantile epileptic encephalopathy, Leigh disease, and secondary pyruvate dehydrogenase complex deficiency. Am. J. Med. Genet. A. 176, 1184-1189.

Tanis, R.M., Piroli, G.G., Day, S.D., and Frizzell, N. (2015). The effect of glucose concentration and sodium phenylbutyrate treatment on mitochondrial bioenergetics and ER stress in 3T3-L1 adipocytes. Biochim. Biophys. Acta 1853, 213-221.

Terburgh, K., Lindeque, Z., Mason, S., van der Westhuizen, F., and Louw, R. (2019). Metabolomics of Ndufs $4^{--}$skeletal muscle: Adaptive mechanisms converge at the ubiquinone-cycle. Biochim. Biophys. Acta Mol. Basis Dis. 1865, 98-106.

Ternette, N., Yang, M., Laroyia, M., Kitagawa, M., O'Flaherty, L., Wolhulter, K., Igarashi, K., Saito, K., Kato, K., Fischer, R., et al. (2013). Inhibition of mitochondrial aconitase by succination in fumarate hydratase deficiency. Cell Rep. 3, 689-700.

Titov, D.V., Cracan, V., Goodman, R.P., Peng, J., Grabarek, Z., and Mootha, V.K. (2016). Complementation of mitochondrial electron transport chain by manipulation of the NAD $/ \mathrm{NADH}$ ratio. Science. 352, 231235.

Yang, Y., and Gibson, G.E. (2019). Succinylation links metabolism to protein functions. Neurochem. Res. 44, 2346-2359.

Yang, L., Shi, Q., Ho, D.J., Starkov, A.A., Wille, E.J., Xu, H., Chen, H.L., Zhang, S., Stack, C.M., Calingasan, N.Y., et al. (2009). Mice deficient in dihydrolipoyl succinyl transferase show increased vulnerability to mitochondrial toxins. Neurobiol. Dis. 36, 320-330.

Zhang, Z., Tan, M., Xie, Z., Dai, L., Chen, Y., and Zhao, Y. (2011). Identification of lysine succinylation as a new post-translational modification. Nat. Chem. Biol. 7, 58-63. 


\section{EXPERIMENTAL MODELS AND SUBJECT DETAILS}

$\underline{\text { Mice }}$

Animal care and use procedures were carried out in accordance with the National Institutes of Health Guide for the Care and Use of Laboratory Animals, and approved by The University of South Carolina Animal Care and Use Committee. Mice were housed in a pathogen-free animal facility under a $12 \mathrm{~h}$ light/dark cycle (lights on from 7AM to 7PM) and received Teklad 8904 rodent diet and water ad libitum. Ndufs4 ${ }^{+/-}$mice (129/Sv x C57BL/6 mixed background) from our colony, originally obtained from Drs. Richard Palmiter and Albert Quintana (Seattle Children's Research Institute, Seattle, WA) (Kruse et al., 2008; Quintana et al., 2010), were mated in trios (one male and two females), switched to a breeder's rodent diet (LabDiet 5058) and the litters were weaned at 21 days of age. Genotyping was performed as previously described (Piroli et al., 2016), and both male and female mice were used. Ndufs $4^{+/+}$and Ndufs4 ${ }^{-}$ 1- mice were sacrificed by decapitation under deep isoflurane anesthesia at 8 weeks of age. The brain was removed from the skull, and the brainstem (BS), olfactory bulbs (OB) and cerebellum were dissected. For some protocols fresh brain regions were used immediately; on other occasions the tissues were snap frozen in liquid nitrogen and stored at $-80^{\circ} \mathrm{C}$ until further use (see METHODS DETAILS).

\section{$\underline{\text { N1E-115 Cells }}$}

N1E-115 cells neuroblastoma cells (subclone N1E-115-1) were expanded in non-differentiation medium containing 90\% DMEM and 10\% FBS, as previously described (Piroli et al., 2016; Piroli et al., 2019). At 80\% confluence, the cells were differentiated into neurons in the presence of $2 \%$ FBS and $1.25 \%$ dimethyl sulfoxide in DMEM for 5 days.

\section{METHOD DETAILS}

\section{Mouse brain preparations for immunoblotting}

Considering the abundance of tubulin in brain preparations, the high level of tubulin succination, and the location of the $\sim 48-50 \mathrm{kDa}$ succinated band that we describe in this manuscript immediately below tubulin, we followed three different protocols in an attempt to enrich the preparations in the $\sim 48-50 \mathrm{kDa}$ band and at the same time deplete them of tubulin.

First, we subjected mouse BS samples to an in vitro tubulin polymerization protocol that we previously described (Piroli et al., 2014). Briefly, frozen mouse BS samples were reduced to a powder with a pestle in a mortar containing liquid nitrogen. The pulverized tissue was then resuspended in cold Mes/glutamate buffer (0.1 M Mes, pH 6.8, containing $0.5 \mathrm{mM} \mathrm{MgCl}$, $1 \mathrm{mM}$ EGTA, $1 \mathrm{M}$ glutamate, $1 \mathrm{mM}$ DTT and a protease inhibitor cocktail), in a volume ratio of 1:1.5 (powder:buffer). The suspension was pulse sonicated at 2 watts using a Model 100 sonic dismembrator (Fisher Scientific) for 5 intervals of $10 \mathrm{~s}$. The total protein homogenate was then centrifuged at $30,000 \times g$ at $4^{\circ} \mathrm{C}$ for $15 \mathrm{~min}$ to generate a pellet (P1) that was analyzed by immunoblotting, and the supernatant (S1) was subjected to microtubule polymerization by addition of $20 \mu \mathrm{M}$ taxol and $1 \mathrm{mM} \mathrm{GTP}$, followed by incubation for $30 \mathrm{~min}$ at $37^{\circ} \mathrm{C}$. 
Following further centrifugation at $30,000 \times \mathrm{g}$ for $30 \mathrm{~min}$ at $37^{\circ} \mathrm{C}$, the supernatant of microtubules (SM) was removed, desalted through Zeba Spin Columns (MW cut-off $7 \mathrm{kDa}$, Thermo Scientific) and analyzed by immunoblotting; the microtubular pellet (MP) composed of $>95 \%$ tubulin was not used.

Second, we prepared gliosomes and synaptosomes from BS and cerebellum samples according to Carney et al. (2014), with minor modifications. Briefly, fresh BS and cerebellum samples were homogenized in $0.32 \mathrm{M}$ sucrose, $1 \mathrm{mM}$ EDTA, $10 \mathrm{mM}$ HEPES pH 7.4 using a glass-teflon homogenizer; the resulting crude homogenates were centrifuged at $1,000 \times g$ at $4^{\circ} \mathrm{C}$ for $5 \mathrm{~min}$. The supernatants (cleared homogenates) were then loaded on top of a gradient composed of layers containing 3, 7, 10 and $20 \%$ Percoll in the homogenization buffer; a fraction of the cleared homogenate was saved for immunoblotting comparative purposes. The gradients were centrifuged at $33,500 \times g$ at $4^{\circ} \mathrm{C}$ for 6 min without brakes, and the interfaces between 3 and $7 \%$ Percoll (gliosomes), 10 and 20\% (synaptosomes) and the loose pellet at the bottom of the tubes were collected and washed with the homogenization buffer to remove the Percoll. All the collected fractions and the cleared homogenates were analyzed by immunoblotting.

Third, we prepared purified mitochondrial fractions according to Kayser et al. (2016), with minor changes. Briefly, fresh BS samples were homogenized in $225 \mathrm{mM}$ mannitol, $75 \mathrm{mM}$ sucrose, $1 \mathrm{mM}$ EGTA, $5 \mathrm{mM}$ HEPES pH 7.2 containing $1 \mathrm{mg} / \mathrm{ml}$ fatty acid-free BSA and a protease inhibitor cocktail using a glass-teflon homogenizer; the resulting crude homogenates were centrifuged at $1,100 \times g$ at $4^{\circ} \mathrm{C}$ for $2 \mathrm{~min}$. The supernatants (cleared homogenates) were added Percoll to a $5 \%$ concentration, and then layered on top of $15 \%$ Percoll; a fraction of the cleared homogenate was saved for immunoblotting comparative purposes. The gradients were centrifuged at $18,500 \times g$ at $4^{\circ} \mathrm{C}$ for $10 \mathrm{~min}$; the top layer, the interface and the lower layer were removed and the loose pellets at the bottom of the tubes were collected and washed with $250 \mathrm{mM}$ sucrose, $0.1 \mathrm{mM}$ EGTA, $5 \mathrm{mM}$ HEPES pH 7.2 to remove the Percoll. The mitochondrial pellets and the cleared homogenate were analyzed by immunoblotting.

\section{Cell Treatments}

To increase succination, N1E-115 cells were treated during the final $24 \mathrm{~h}$ of differentiation with 0-100 $\mu \mathrm{M}$ dimethyl fumarate (DMF) prepared in Dulbecco's PBS (DPBS) and filtered; some of the wells received dimethyl succinate (DMS) in the same range of concentrations as a negative control. The cell collection and preparation is described in detail in the section "Measurement of the $\alpha$-ketoglutarate dehydrogenase complex activity".

\section{One-dimensional PAGE and Western Blotting}

Western blotting was performed as described previously (Piroli et al., 2014; Piroli et al., 2016; Piroli et al., 2019). Ten to fifty $\mu \mathrm{g}$ of proteins were run on $12 \%$ gels (Criterion, BioRad) and transferred to PVDF membranes. Immunoblotting was performed with antibodies listed in the Key Resources Table. The preparation of the polyclonal anti-2SC antibody has been described previously (Nagai et al., 2007). In most cases, membranes were stripped with $62.5 \mathrm{mM}$ Tris, $\mathrm{pH} 6.8$, containing $2 \%$ SDS and $0.7 \%$ 2mercaptoethanol for $15 \mathrm{~min}$ at $65^{\circ} \mathrm{C}$ prior to reprobing. Chemiluminescent signals were captured on photographic film (HyBlot $\mathrm{CL}$ ). 
$\underline{\text { Two-dimensional Gel Electrophoresis and Western Blotting }}$

Isoelectric focusing on pl 4-7, $11 \mathrm{~cm}$ strips and two-dimensional (2D) gel electrophoresis was performed in an Ettan IPGphor II device (Amersham Biosciences) as described previously (Piroli et al., 2014; Piroli et al., 2016). The 2D gels were transferred onto PVDF membranes to detect protein succination by western blotting; the blots were then stripped and subjected to DLST detection.

Measurement of the $\alpha$-ketoglutarate dehydrogenase complex activity in brain regions

The activity of the $\alpha$-ketoglutarate dehydrogenase complex (KGDHC) was measured by following the formation of NADH at $340 \mathrm{~nm}$ according to Yang et al. (2009), with minor modifications. BS and OB frozen samples were homogenized in $50 \mathrm{mM}$ Tris- $\mathrm{HCl} \mathrm{pH} 7.2$ containing $1 \mathrm{mM}$ dithiothreitol, $0.2 \mathrm{mM} \mathrm{EGTA}, 0.4 \%$ Triton X-100 and a protease inhibitor cocktail using a glass-teflon homogenizer. The crude homogenates were centrifuged at $2,000 \times g$ for $4 \mathrm{~min}$ at $4^{\circ} \mathrm{C}$; the supernatants were used to determine KGDHC activity. One hundred $\mu \mathrm{l}$ of a reaction mix containing $50 \mathrm{mM}$ Tris- $\mathrm{HCl} \mathrm{pH} 7.8,1 \mathrm{mM} \mathrm{MgCl}, 1 \mathrm{mM} \mathrm{CaCl}, 0.5 \mathrm{mM}$ EDTA, $0.3 \mathrm{mM}$ thiamine pyrophosphate, $0.1 \%$ Triton X-100 and $1 \mathrm{mM}$ dithiothreitol were added to the wells of a 96-well plate, followed by $50 \mu \mathrm{l}$ of an assay mix containing $50 \mathrm{mM}$ Tris- $\mathrm{HCl} \mathrm{pH} \mathrm{7.8,} 3 \mathrm{mM} \mathrm{NAD}^{+}$ and $0.75 \mathrm{mM}$ coenzyme $A$, and then $25 \mu \mathrm{l}$ of the tissue preparations containing 5-10 $\mu$ g protein (or homogenization buffer for the blanks). A baseline reading every min for $10 \mathrm{~min}$ with the temperature set at $30^{\circ} \mathrm{C}$ was taken at $340 \mathrm{~nm}$ in a plate reader (Tecan Safire ${ }^{2}$, TECAN Systems Inc.). After the addition of $25 \mu \mathrm{l} 3 \mathrm{mM} \alpha$-ketoglutarate in $50 \mathrm{mM}$ Tris- $\mathrm{HCl} \mathrm{pH} 7.8$ (or $50 \mathrm{mM}$ Tris- $\mathrm{HCl} \mathrm{pH} 7.8$ for "no substrate" controls), further readings were taken for an additional period of $60 \mathrm{~min}$. The readings in the absence of $\alpha$-ketoglutarate were subtracted from those in the presence of the substrate, and these corrected values were plotted as a function of time to calculate the $\mathrm{V}_{\max }$ from the slopes. Each sample was run in triplicates that were averaged; the $\mathrm{n}$ size was 3-4 for each experimental group. The results were converted to $\mathrm{mU} / \mathrm{mg}$ of protein considering $\mathrm{E}_{\mathrm{NAD}(340)}=6.22 \mathrm{~cm}^{-1} \mathrm{mM}^{-1}$ and a path length of $0.6 \mathrm{~cm}$.

Measurement of the KGDHC activity in purified bovine heart complex and N1E-115 cells

In vitro succination effects on the activity of a commercially available bovine heart KGDHC standard was studied after incubation for $18 \mathrm{~h}$ at room temperature with a range of 0-2 $\mathrm{mM}$ DMF following the protocol described above for brain regions; dithiothreitol was replaced with $300 \mu \mathrm{M}$ tris(2-carboxyethyl) phosphine (TCEP) to avoid the possible reaction of DMF with dithiothreitol. The results of the KGDHC activity were expressed as \% of the control group (no DMF added). The KGDHC activity was also measured in N1E-115 cells treated with DMF or DMS (see "Cell Treatments" above) with minor modifications. Briefly, the medium was removed; the cells were rinsed 3 times with DPBS and gently scraped with $50 \mathrm{mM}$ Tris- $\mathrm{HCl}$ pH 7.2 containing $1 \mathrm{mM}$ dithiothreitol, 0.2 mM EGTA, $0.4 \%$ Triton X-100 and a protease inhibitor cocktail. The extracts were homogenized in a glass-glass homogenizer, the resulting homogenates were centrifuged at 2,000 $\times \mathrm{g}$ for $4 \mathrm{~min}$ at $4^{\circ} \mathrm{C}$, and the supernatants were used to determine KGDHC activity following the protocol described above for brain regions. The results of the KGDHC activity were expressed as \% of the control group (no DMF or DMS added).

Substrate level phosphorylation measurements 
Mitochondrial isolation and measurement of ATP synthesis was performed according to Komlódi and Tretter (2017), with minor modifications. Freshly obtained BS and OB from Ndufs4 KO mice and WT littermates were immediately homogenized in $5 \mathrm{mM}$ Tris pH 7.4 containing $225 \mathrm{mM}$ mannitol, $75 \mathrm{mM}$ sucrose and $1 \mathrm{mM}$ EGTA using a glass-teflon homogenizer. The initial homogenates were centrifuged at $1,300 \times g$ for $3 \mathrm{~min}$, and the supernatant was further centrifuged at 20,000 $\mathrm{g}$ for $10 \mathrm{~min}$. The resultant pellet was resuspended in $15 \%$ Percoll and then layered on top of a discontinuous Percoll gradient consisting of $23 \%$ and $40 \%$ layers. The gradients were then centrifuged at $30,700 \times g$ for 8 min using no brake at the end. Immunoblotting experiments (data not shown) showed that the interface between the $23 \%$ and $40 \%$ Percoll and the loose pellet in the $40 \%$ Percoll layer were enriched in mitochondrial markers and devoid of myelin. Consequently, these two fractions were pooled together, topped with homogenization buffer and centrifuged for $10 \mathrm{~min}$ at 16,600 $\mathrm{g}$; and the pellet was re-suspended in homogenization buffer and subsequently centrifuged again for $10 \mathrm{~min}$ at 6,300 $\mathrm{xg}$ for $10 \mathrm{~min}$. The resulting purified mitochondrial pellet was re-suspended in $5 \mathrm{mM}$ Tris pH 7.4 containing $225 \mathrm{mM}$ mannitol and $75 \mathrm{mM}$ sucrose, and immediately used for mitochondrial ATP synthesis measurements. The assay medium consisted of $20 \mathrm{mM}$ HEPES pH 7.0 containing $0.1 \mathrm{mM} \mathrm{EGTA}, 125 \mathrm{mM} \mathrm{KCl}, 2 \mathrm{mM} \mathrm{K}_{2} \mathrm{HPO}_{4}, 1 \mathrm{MgCl}_{2}$, and $0.025 \%$ fatty acid-free bovine serum albumin, with the addition of $3 \mathrm{mM} \mathrm{NADP}+5 \mathrm{mM}$ glucose, 300 $\mu$ M AP5 ( $\mathrm{P}^{1}, \mathrm{P}^{5}$-Di(adenosine-5') pentaphosphate; an inhibitor of adenylate kinase), $0.75 \mathrm{U} / \mathrm{ml}$ hexokinase, and $0.25 \mathrm{U} / \mathrm{ml}$ glucose-6-phosphate dehydrogenase, in a final volume of $200 \mu \mathrm{l}$. ATP formation was detected through the phosphorylation of glucose by added hexokinase, coupled to the oxidation of the resulting glucose 6-phosphate by added glucose 6-phosphate dehydrogenase, with formation of NADPH. NADPH synthesis was followed at $340 \mathrm{~nm}$ in a plate reader as described for the measurement of $\alpha$ ketoglutarate dehydrogenase complex activity (see above). Basal measurements started upon addition of the mitochondrial samples $\left(5 \mu \mathrm{g} /\right.$ well) and ADP $(2 \mathrm{mM})$ for $10 \mathrm{~min}$ at $37^{\circ} \mathrm{C}$, followed by the addition of $\alpha$ ketoglutarate or succinate (both at $5 \mathrm{mM}$ ); some wells also received oligomycin $(8 \mu \mathrm{M})$ to determine total ATP synthesis (oxidative phosphorylation + substrate level phosphorylation) in the absence of oligomycin, and substrate level phosphorylation in the presence of oligomycin. Corrections in the absence of substrate were applied, and these corrected values were plotted as a function of time to calculate the ATP synthesis from the slopes. Each sample was run in triplicates that were averaged; the $\mathrm{n}$ size was 7-12 for each experimental group. The results were converted to nmoles ATP/min/mg of protein using an ATP curve (range: 0-20 nmoles). These mitochondrial fractions were also used for immunoblotting experiments (succinylation, acetylation and succination studies in Figure 4)

\section{Determination of Protein Content}

The protein content in all the brain and cell preparations, including samples for immunoblotting and those for the determinations of enzymatic activity was determined by the method of Lowry et al. (1951).

\section{Protein identification by liquid chromatography-tandem mass spectrometry (LC-MS/MS)}

The in-gel protein digestion method used was previously described (Piroli et al., 2016; Piroli et al., 2019). Briefly, samples of the pellets obtained from the synaptosomal preparations ( $20 \mu \mathrm{g}$ of protein) were resolved by SDS-PAGE in 7.5\% gels; the gels were stained with Coomassie Brilliant Blue and the bands of interest in the $\sim 48-50 \mathrm{kDa}$ region were excised. The gel pieces were destained, washed with $50 \mathrm{mM}$ 
ammonium bicarbonate in $50 \%$ acetonitrile, and dehydrated with $100 \%$ acetonitrile; proteins were then reduced with $10 \mathrm{mM}$ dithiothreitol and alkylated with $170 \mathrm{mM}$ 4-vinylpyridine. Protein digestion was carried out overnight at $37^{\circ} \mathrm{C}$ in the presence of $500 \mathrm{ng}$ sequencing grade modified trypsin in $50 \mathrm{mM}$ ammonium bicarbonate. After gel extraction with $5 \%$ formic acid in 50\% acetonitrile, the samples were analyzed in a blinded manner on a Dionex Ultimate 3000-LC system (Thermo Scientific) coupled to a Velos Pro Orbitrap mass spectrometer (Thermo Scientific). The LC solvents were $2 \%$ acetonitrile/0.1\% formic acid (Solvent A) and $80 \%$ acetonitrile/0.1\% formic acid (Solvent B); the water used for these solvents was LC-MS grade. Peptides were first trapped on a $2 \mathrm{~cm}$ Acclaim PepMap-100 column (Thermo Scientific) with Solvent $A$ at $3 \mu \mathrm{l} / \mathrm{min}$. At $4 \mathrm{~min}$ the trap column was placed in line with the analytical column, a $75 \mu \mathrm{m}$ C18 stationary-phase LC PicoChip Nanospray column (New Objective). The peptides were eluted with a gradient from $98 \% \mathrm{~A}: 2 \% \mathrm{~B}$ to $40 \% \mathrm{~A}: 60 \% \mathrm{~B}$ over $30 \mathrm{~min}$, followed by a $5 \mathrm{~min}$ ramp to $10 \% \mathrm{~A}: 90 \% \mathrm{~B}$ that was held for $10 \mathrm{~min}$. The Orbitrap was operated in data-dependent acquisition MS/MS analysis mode and excluded all ions below 200 counts. Following a survey scan (MS1), up to 8 precursor ions were selected for MS/MS analysis. All spectra were obtained in the Orbitrap at 7500 resolution. The DDA data were analyzed using Proteome Discover 1.4 software with SEQUEST algorithm against the uniprot_ref_mouse database (2014-10-03 version, 52,474 proteins) with XCorr validation $>2(+2)$ or $>2.5(+3)$. An allowance was made for 2 missed cleavages following trypsin digestion. No fixed modifications were considered. The variable modifications of methionine oxidation $\left(\mathrm{M}^{\mathrm{Ox}}\right)$, proline hydroxylation $\left(\mathrm{P}^{\mathrm{Ox}}\right)$, cysteine pyridylethylation $\left(C^{P E}, 105.058\right)$ or cysteine succination by fumarate $\left(C^{2 S C}, 116.011\right)$ were considered with a mass tolerance of $15 \mathrm{ppm}$ for precursor ions and a mass tolerance of $10 \mathrm{ppm}$ for fragment ions. The results were filtered with a false discovery rate of 0.01 . For all DLST peptides identified the spectra were manually inspected to confirm the abundance of product ions. The software Scaffold 4D was used to generate the spectrum in Figure 1D.

\section{Quantification and Statistical Analysis}

Results were expressed as means \pm standard error of the mean (SEM). For the enzymatic activities, a description of the calculations is presented in the corresponding subheading under METHOD DETAILS. Statistical comparisons between two experimental groups were performed using unpaired $t$ tests; when three or more groups were compared, one-way ANOVA followed by Student-Neuman-Keuls' test was applied. The $\mathrm{n}$ size for each particular experiment is described in the Figure legends, and represents the number of individual biological replicates. Differences were considered statistically significant when a $P$ value $<0.05$ was achieved. The software Image $\mathrm{J}(\mathrm{NIH})$ was used for the quantification of band intensities by densitometry (Tanis et al., 2015). The software GraphPad Prism V5.0 was used for statistical analysis and graphs.

\section{$\underline{\text { Data and Code Availability }}$}

The mass spectrometry .RAW files used to identify dihydrolipoyllysine-residue succinyltransferase peptides have been deposited in Mendeley Data with the identifier:

http://dx.doi.org/10.17632/3y7yp7c8bj.1 


\section{Figure Legends}

Figure 1: Identification of dihydrolipoyllysine-residue succinyltransferase (DLST) as a succinated protein in the brainstem (BS) of the Ndufs4 KO mouse.

$A$, Detection of a distinct succinated band at $\sim 48-50 \mathrm{kDa}$ (arrow in $2 \mathrm{SC}$ panel) was limited to pellets obtained after high speed centrifugation of Ndufs4 KO BS homogenates. $B$, Homogenates, gliosomes, synaptosomes, and a pellet fraction obtained from BS were analyzed for $\alpha$-tubulin, GFAP, synaptophysin and VDAC2 to characterize the purity of each fraction. Removal of tubulin in the pellet fractions allowed for the clear detection of a $\sim 48-50 \mathrm{kDa}$ succinated band in the KO lanes (arrow in 2SC panel). C, Analysis of homogenates and purified mitochondrial preparations showed an increase of a succinated band at $\sim 48$ $50 \mathrm{kDa}$ in $\mathrm{KO}$ mitochondrial lanes (arrow), which overlapped with DLST detection. $D, \mathrm{MS} / \mathrm{MS}$ spectrum of the peptide NVETM ${ }^{\text {ox }}$ NYADIER corresponding to DLST in a $\sim 48-50 \mathrm{kDa}$ band isolated from the pellet preparation used in B. E, Increased succination of Ndufs 4 KO BS preparations for a series of spots at $\sim 48$ $50 \mathrm{kDa}$ and $\mathrm{pH}$ 5.5-6.3 (rectangular area, 2SC KO panel). Arrows denote co-localization of succinated spots with DLST-immunoreactive spots (2SC and DLST KO panels). For the WT BS, succinated spots did not colocalize with the DLST spots. $F$, Total DLST and $\alpha$-tubulin content in BS homogenates of WT and Ndufs4 KO mice. $G$, Quantification of the blots in $F$ showed no significant differences in normalized DLST expression between WT and KO BS ( $n=3)$.

Molecular weight markers are shown on the left.

In $A, B$ and $F$, Coomassie staining was used to verify even load of the lanes.

Figure 2: The $\alpha$-ketoglutarate dehydrogenase complex (KGDHC) activity is reduced as a consequence of fumarate derived protein succination.

$A$, Fumarate content is increased in the OB of Ndufs4 KO mice (***p<0.001 vs. WT; $n=4-5) . B-C$, KGDHC activity is reduced in the $B S(B)\left({ }^{*} p<0.05 ; n=3-4\right)$, and $O B(C)\left({ }^{*} p<0.01 ; n=4\right)$ of Ndufs 4 KO vs. WT mice. $D$, Incubation with dimethyl fumarate (DMF) decreases the activity of a bovine heart KGDHC standard in a dose-dependent manner ( ${ }^{* * *} p<0.001$ vs. $0 \mathrm{mM} \mathrm{DMF,}{ }^{\#} p<0.01$ vs. $0.5 \mathrm{mM} \mathrm{DMF;} n=3$ ). E, Addition of DMF to differentiated N1E-115 neurons decreases KGDHC activity, whereas dimethyl succinate (DMS) does not decrease the activity ( ${ }^{* *} p<0.01,{ }^{* * *} p<0.001$ vs. control; $n=3-5$ ). $F$, Protein succination increases in N1E-115 neurons exposed to DMF. Two bands, one at $\sim 48-50 \mathrm{kDa}$ (arrow) and another at $68-70 \mathrm{kDa}$ showed a dose response increase in succination. DLST immunoreactivity co-localized with the succinated band at $\sim 48-50 \mathrm{kDa}$. Molecular weight markers are shown on the left side.

Figure 3: Total ATP synthesis and substrate level phosphorylation (SLP) are decreased in the olfactory bulb (OB) of the Ndufs4 KO mouse.

$A-B$, ATP synthesis in the $\mathrm{OB}(A)$ and the $\mathrm{BS}(B)$ in the presence of $5 \mathrm{mM}$-ketoglutarate ( $\alpha \mathrm{KG})$ as respiratory substrate, with or without the addition of $8 \mu \mathrm{M}$ oligomycin (O). The bars without oligomycin represent total ATP synthesis (OXPHOS + SLP), and the bars with oligomycin represent SLP. A decrease in total ATP synthesis and SLP is present in the OB of the KO mice ( ${ }^{*} p<0.05$ vs. WT; $n=5-6$ for $O B, n=4$ for $B S$ ). 
$C-D$, Same as in $A-B$ but using $5 \mathrm{mM}$ succinate (SUC) as respiratory substrate. No differences were observed between WT and Ndufs4 KO mice in this condition ( $n=9-12$ for $O B, n=7$ for BS).

Figure 4: Succinylation of mitochondrial proteins is reduced in the BS of the Ndufs4 KO mouse.

$A$, A general decrease in protein succinylation was observed in mitochondrial fractions obtained from BS of KO mice. $B$, Quantification of the most prominent bands in $A$ (arrowheads) confirmed a significant decrease in protein succinylation in mitochondrial fractions obtained from the BS of Ndufs 4 KO mice $\left({ }^{* * *} p<0.001\right.$ vs. WT; $\left.n=8\right) . C$, Increased protein acetylation was observed in mitochondrial fractions obtained from BS of KO mice. $D$, Quantification of the most prominent bands in $C$ (arrowheads) confirmed a significant increase in band 1 acetylation in mitochondrial fractions obtained from the BS of Ndufs4 KO mice ( ${ }^{*} p<0.05$ vs. WT; $n=8$ ). In $A$ and $C$, blots were stripped after the initial probing and re-probed with an anti-fumarase antibody for normalization, followed by staining with Coomassie Brilliant Blue. Molecular weight markers are shown on the right side in $A$ and $C$. 
bioRxiv preprint doi: https://doi.org/10.1101/2020.01.09.900514; this version posted January 9, 2020. The copyright holder for this preprint (which was not certified by peer review) is the author/funder, who has granted bioRxiv a license to display the preprint in perpetuity. It is made available under aCC-BY-NC-ND 4.0 International license.

Figure 1

A

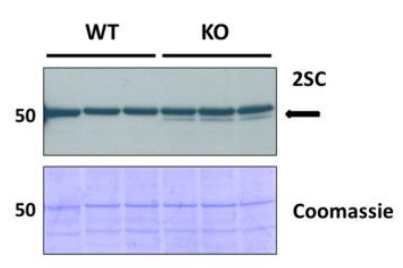

C

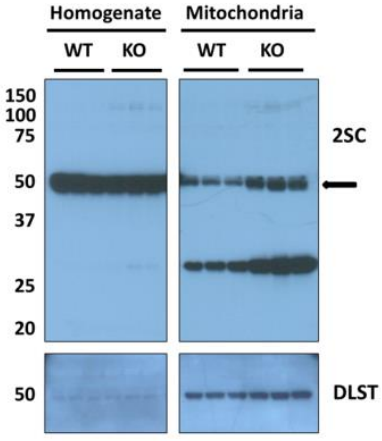

B

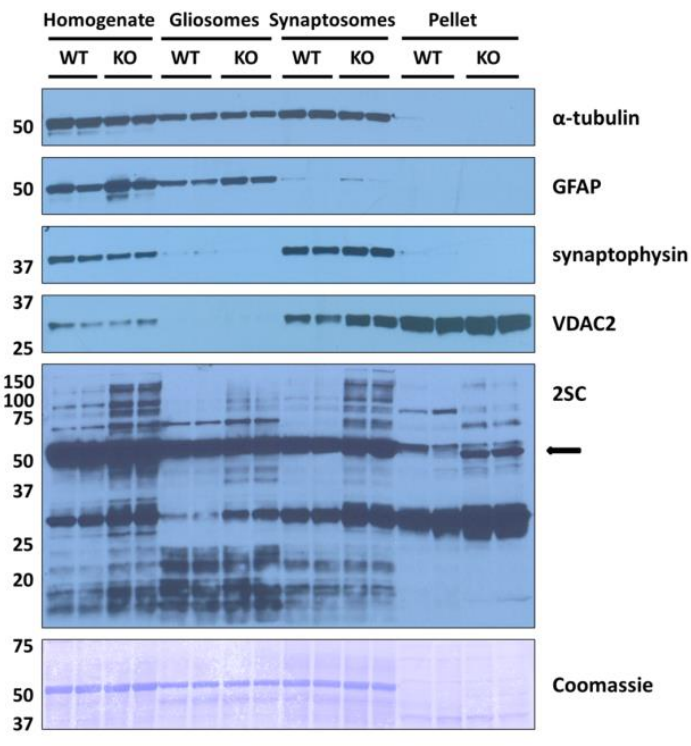

D

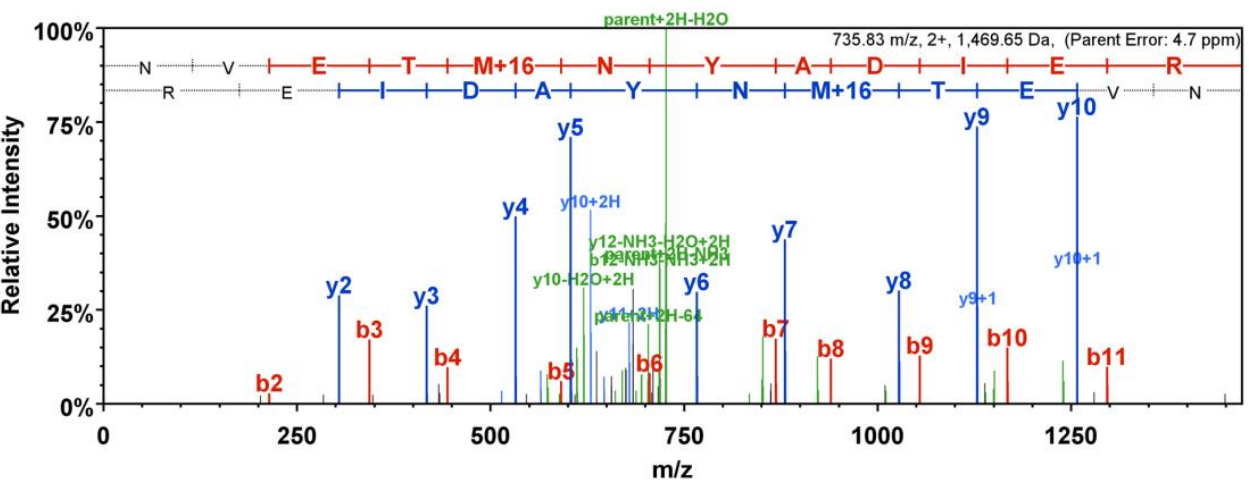

E
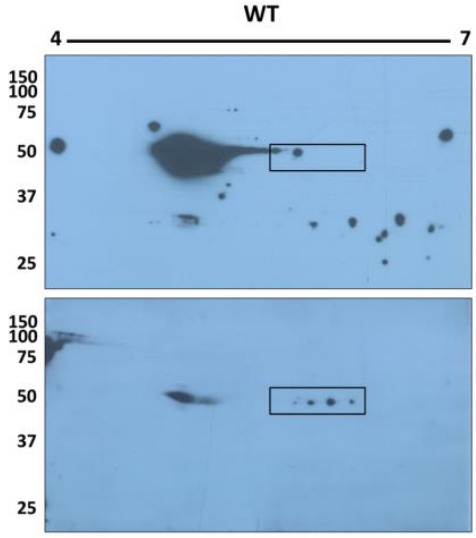

ко

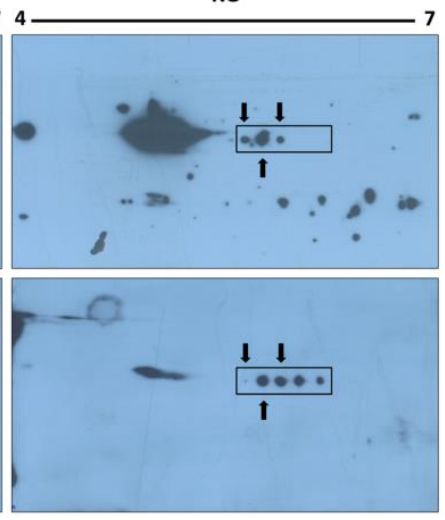

F

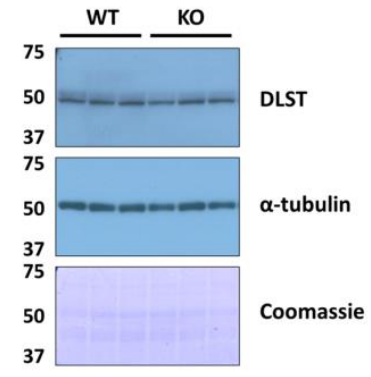

DLST

$2 \mathrm{sC}$
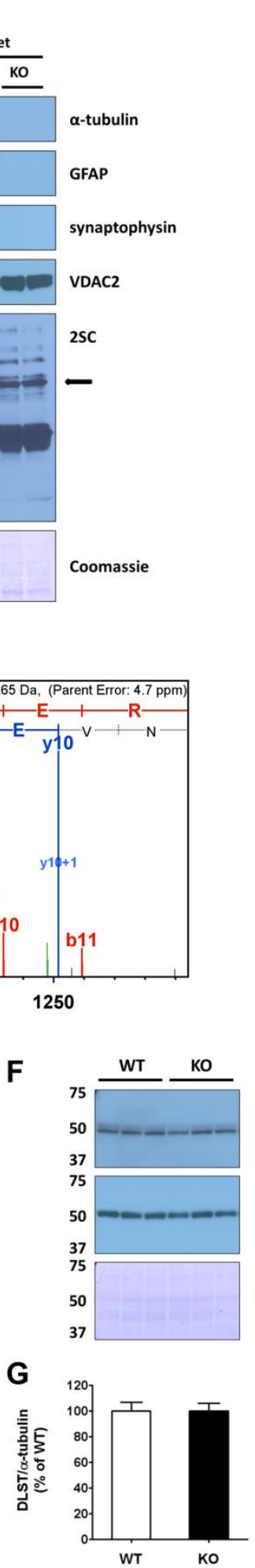
Figure 2

A

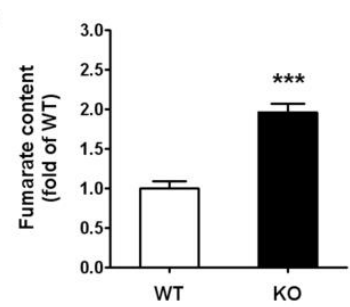

D

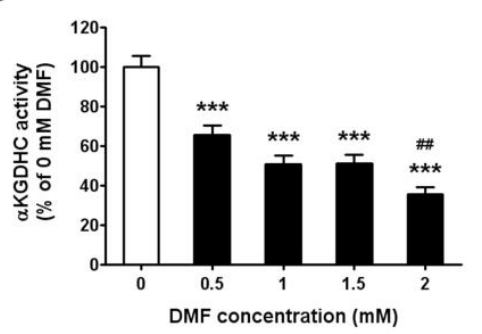

B

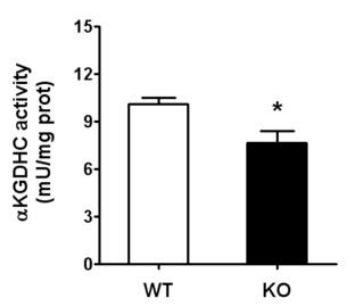

$\mathbf{E}$

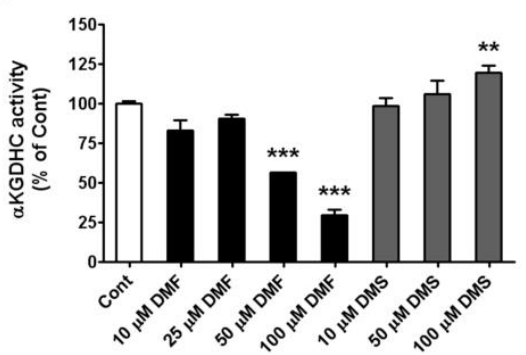

C

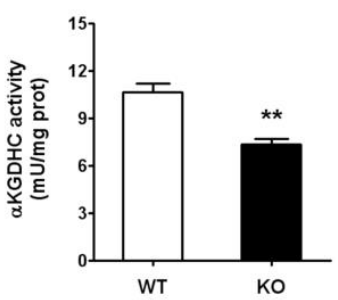

F

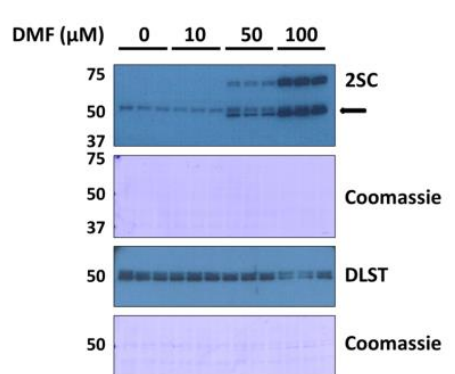


Figure 3

\section{Olfactory Bulb}

A

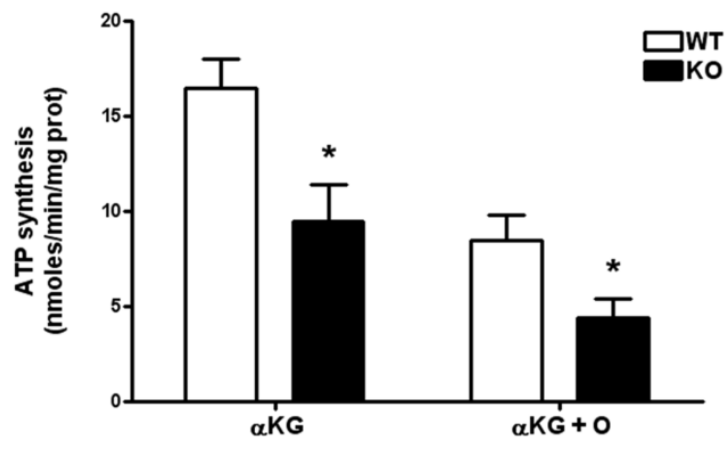

C

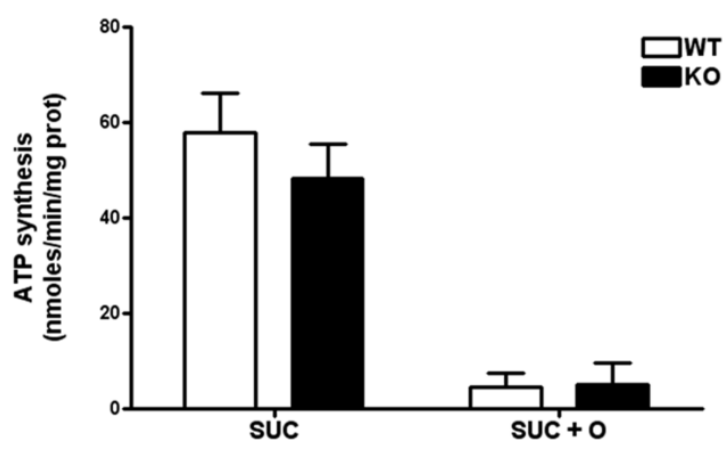

Brainstem

B

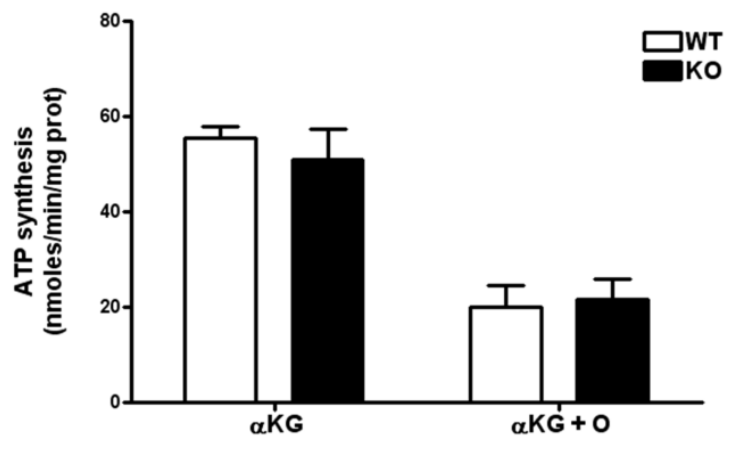

D

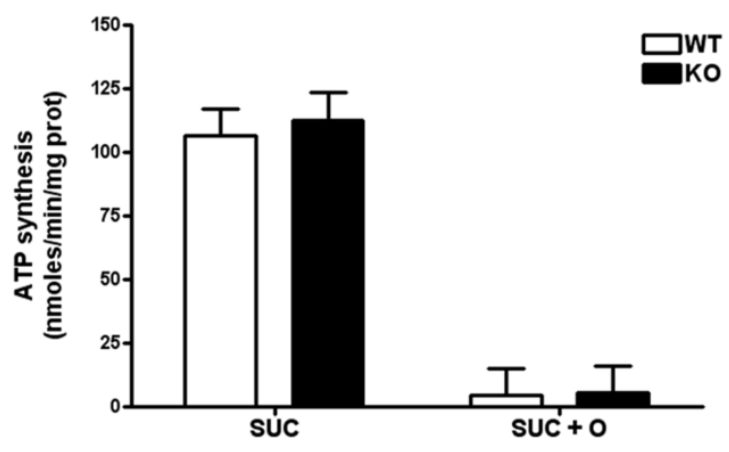


bioRxiv preprint doi: https://doi.org/10.1101/2020.01.09.900514; this version posted January 9,2020 . The copyright holder for this preprint (which was not certified by peer review) is the author/funder, who has granted bioRxiv a license to display the preprint in perpetuity. It is made available under aCC-BY-NC-ND 4.0 International license.

Figure 4

A

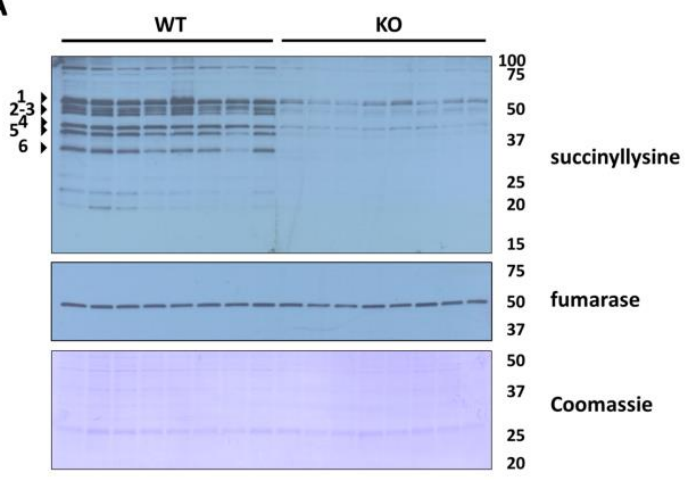

C

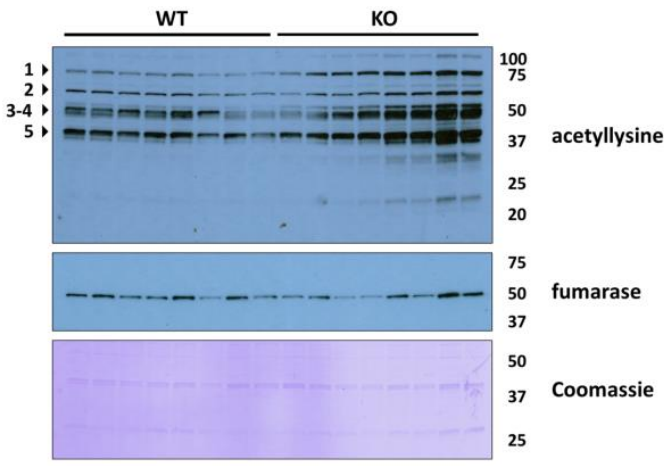

B

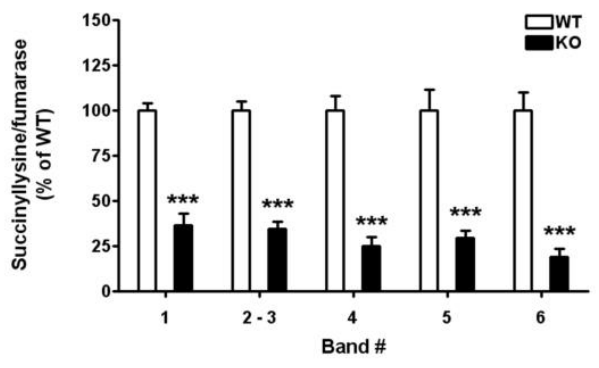

D

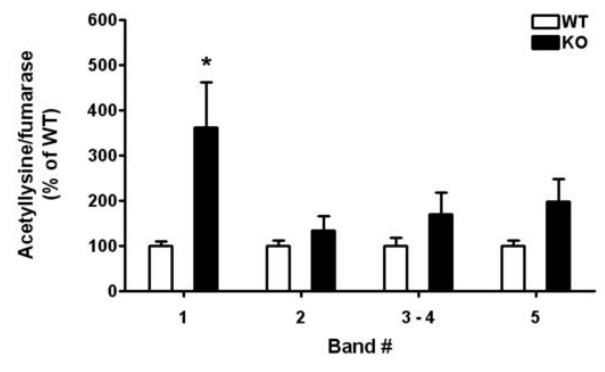




\section{Supplemental Material}

A

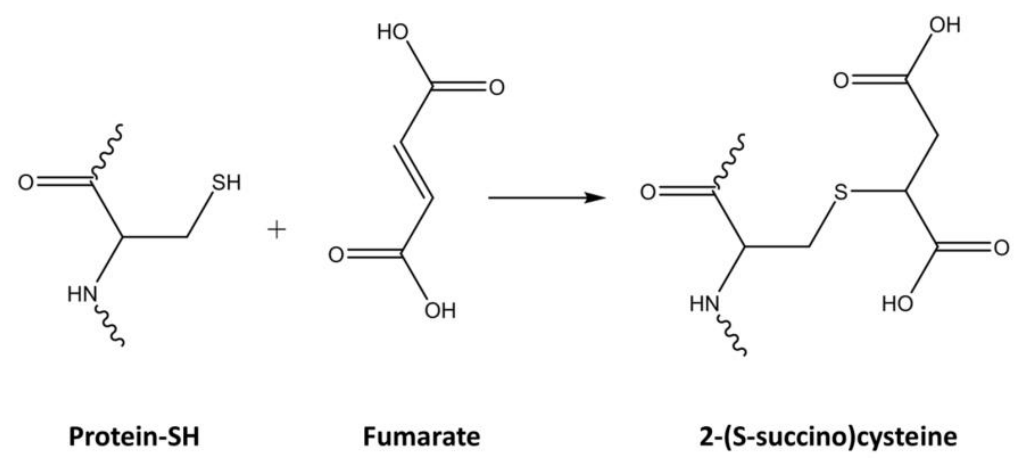

B

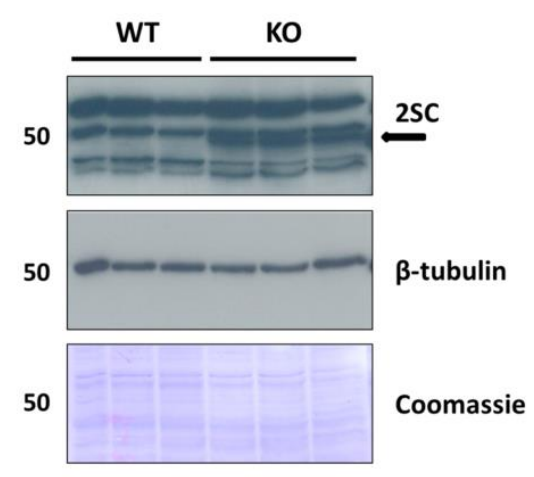

\section{C}

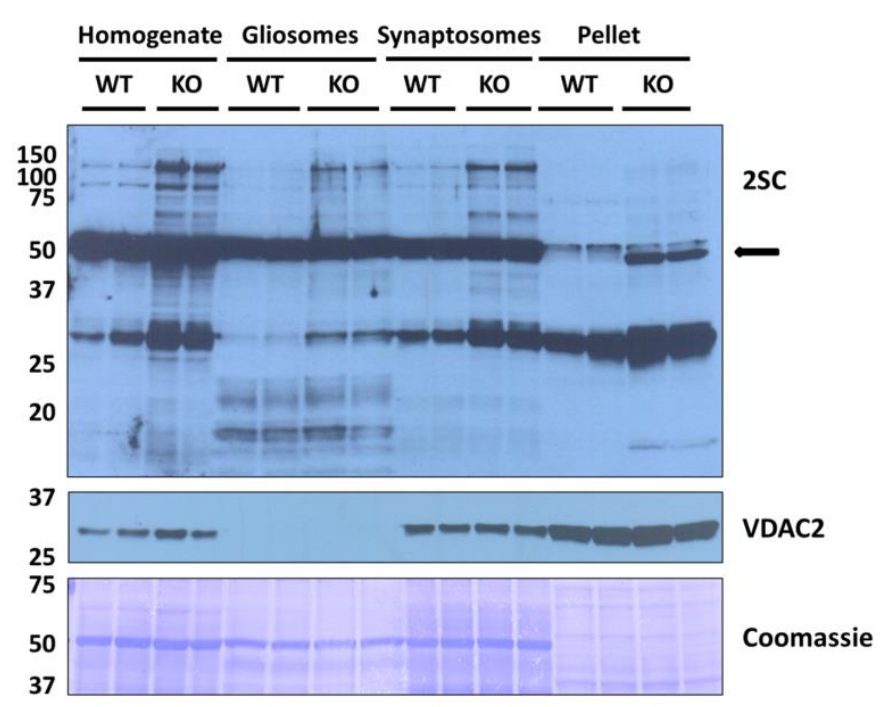

Figure S1: $\mathrm{A}$ band at $\sim 48-50 \mathrm{kDa}$ is differentially succinated in the $\mathrm{BS}$ and $\mathrm{CB}$ of the Ndufs4 KO mouse. $A$, Formation of 2-(S-succino)cysteine (2SC) by reaction of fumarate with cysteine residues in proteins. $B, A$ high speed supernatant after isolation of in vitro polymerized microtubules shows a succinated band at $\sim 48-50 \mathrm{kDa}$ in the BS of the Ndufs4 KO mouse, but not in WT littermates (arrow). Tubulin and Coomassie panels show even load of proteins ( $30 \mu \mathrm{g}$ prot/lane). C Similar to the BS (see Figure $1 \mathrm{~B}$ ), a band at $\sim 48-50$ kDa shows increased succination in a pellet fraction after synapotosomal and gliosomal preparation of the cerebellum of the Ndufs4 KO mouse (arrow in 2SC panel). Further probing after stripping showed an enrichment of VDAC (mitochondrial marker, VDAC panel) in the pellet fraction. Coomassie panel shows even protein load (15 $\mu \mathrm{g}$ prot/lane) within each fraction group. Molecular weight markers are shown on the left side. 


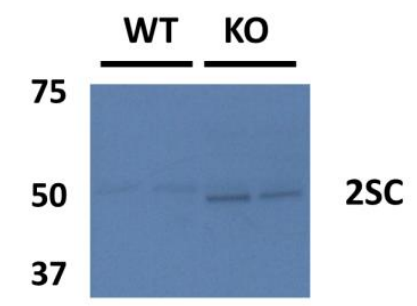

Figure S2: In the BS of Ndufs4 KO but not in WT mice, a band at $\sim 48-50 \mathrm{kDa}$ was succinated in the same mitochondrial fractions used for Figure 4. Protein load was $10 \mu \mathrm{g}$ per lane, and molecular weight markers are shown on the left side.

Table S1: MS/MS peptide identification for dihydrolipoyllisine-residue succinyltransferase component of 2-oxoglutarate dehydrogenase complex. Raw data were searched with the Sequest HT node of Proteome Discover 1.4 (SP1), using the uniprot_ref_mouse database. Variable modifications of methionine oxidation $\left(\mathrm{M}^{\mathrm{OX}}\right)$, proline oxidation $\left(\mathrm{P}^{\mathrm{OX}}\right)$, cysteine pyridylethylation $\left(\mathrm{C}^{\mathrm{PE}}\right)$, and cysteine succination by fumarate $\left(\mathrm{C}^{2 \mathrm{SC}}\right)$ were considered. The peptides identified are reported from 3 individual KO mice analyzed.

\begin{tabular}{|c|c|c|c|c|c|c|}
\hline $\begin{array}{l}\text { Swiss-Prot } \\
\text { Accession \# }\end{array}$ & Protein Identity & \% Coverage & Peptides Identified & PSMs & $\mathrm{M}+(\mathrm{Da})$ & Charge \\
\hline \multirow[t]{10}{*}{ Q9D2G2 } & Dihydrolipoyllisine-residue & 31.87 & VEVRP $^{\mathrm{OX}} \mathrm{M}^{\mathrm{OX}} \mathrm{MYVALTYDHR}$ & 42 & 2011.97898 & 3 \\
\hline & succinyltransferase component of $2-$ & & ASAFALQEQPVVNAVIDDATK & 12 & 2187.14591 & 3 \\
\hline & oxoglutarate dehydrogenase complex & & LGFM $^{\mathrm{OX}}$ SAFVK & 9 & 1015.53142 & 2 \\
\hline & (DLST) (Mus musculus) & & VEGGTPLFTLR & 9 & 1189.66350 & 2 \\
\hline & & & NVETM ${ }^{\mathrm{OX}}$ NYADIER & 8 & 1470.65727 & 2 \\
\hline & & & NVETMNYADIER & 1 & 1454.66118 & 2 \\
\hline & & & DYIDISVAVATPR & 8 & 1419.75774 & 2 \\
\hline & & & TINELGEK & 6 & 903.48235 & 2 \\
\hline & & & NDVITVQTPAFAESVTEGDVR & 6 & 2248.11650 & 2 \\
\hline & & & AKPAETPAPAHK & 3 & 1217.66919 & 3 \\
\hline
\end{tabular}

